\title{
Analysis of Power Consumption Efficiency on Various IoT and Cloud-Based Wireless Health Monitoring Systems: A Survey
}

\author{
Beny Nugraha \\ Departement of Electrical Engineering - Graduate Program, Mercu Buana University, Jakarta, Indonesia \\ E-mail: benynugraha@mercubuana.ac.id \\ Irawan Ekasurya, Gunawan Osman, Mudrik Alaydrus \\ Departement of Electrical Engineering - Graduate Program, Mercu Buana University, Jakarta, Indonesia \\ E-mail: irawan.ekasurya@gmail.com; gunawan.osman@gmail.com; mudrikalaydrus@mercubuana.ac.id
}

\begin{abstract}
Nowadays, various Wireless Health Monitoring Systems use Internet of Things to transmit patient's data over Wireless Sensor Network and then the data is stored and processed via Cloud Computing, however, the use of different kind of Wireless Sensor on each system leads to power efficiency problem. This paper analyses and compares the consumption of power on six Wireless Health Monitoring Systems, which are invented to monitor the patient's condition and transfer the data using Wireless Sensor Network. Three different techniques are analyzed, namely GPRS/UMTS (used in one WHMS), Wi-Fi (used in one WHMS), and Bluetooth (used in four WHMS). This paper concludes that the systems that use Bluetooth as their transmission medium are more effective in reducing power consumption than the other systems that use GPRS/UMTS or Wi-Fi.
\end{abstract}

Index Terms-Cloud Computing, Power Efficiency, Internet of Thing, Wireless Sensor Network, Wireless Health Monitoring Systems.

\section{INTRODUCTION}

The Wireless Health Monitoring Systems (WHMS) can be a solution to provide reliable and low-cost monitoring system that is able to monitor the patient's data in real time for 24 hours a day. WHMS needs a communication medium between its sensor and mobile devices to transmit the patient's data. WHMS can use Bluetooth, Wi-Fi (Wireless-Fidelity), or GPRS (General Packet Radio Service)/UMTS (Universal Mobile Telecommunications System) as its transmission medium

The WHMS works by obtaining, processing, and displaying the patient's biological and physiological data. WHMS that can perform tasks automatically can lessen the patient's recovery time that require continuous monitoring and treatment procedures.

Various WHMS have been made to support patient health monitoring system on both distant and close range by monitoring the heart rate, blood pressure, body temperature and the vital signs of patients of all ages.
Designing cloud-based applications and health monitoring services that are effective and efficient in terms of power consumption is a very big challenge. This challenge motivates us to continue researching in this area, therefore, for the initial work of our big project, in this paper the power efficiency of several WHMS is discussed and compared since each system uses different medium to transmit the patient's data.

The rest of this paper is organized as the following: Brief description of related technologies such as wireless sensor networks, Cloud Computing, Internet of Things (IoT), Bluetooth, Wi-Fi, and GPRS/UMTS are discussed in Section II. In Section III, six WHMS, namely Ubiquitous Mobile Health Monitoring System for Elderly (UMHMSE), Sensing Health with Intelligence Modularity, Mobility and Experimental Reusability (SHIMMER)-based Windows mobile system, MultiHopping Wireless Body Area Network system (WBAN), Hybrid mobile cloud based ECG monitoring system, Bluetooth Low Energy (BLE)-based wireless electrocardiogram monitoring system, and OSAS phonebased e-health system are described. In Section IV, the comparison analysis of those six WHMS is presented. The conclusion of this paper is given in Section V and lastly, our future work is presented in Section VI.

\section{RELATED TECHNOLOGIES}

This Section defines the communication tehcnologies that are used by the WHMSs that are surveyed in this paper, these technologies are the Wireless Sensor Network (WSN), Cloud Computing, IoT, Bluetooth, Wi$\mathrm{Fi}$, and GPRS/UMTS.

\section{A. Wireless Sensor Network (WSN)}

WSN is used as a new solution that is able to collect information through radio spectrum for a variety of different applications, such as transportation, business, health, industrial, and environmental monitoring [1]. Collecting patient physiological parameters using wireless data transmission of multi-channel high 
frequency can improve hospital management information system that is able to create a modern health monitoring system in real-time. WSN has a big advantage in terms of deployment of sensor devices, which are small, low-cost and can be used anywhere [2].

\section{B. Cloud Computing}

Cloud Computing technology is designed by the National Institute of Standards and Technology (NIST) [3] to increase the capacity of joint computing resources that is fast and safe at various locations around the world. Cloud Computing is a technology resource sharing and collection of data among users through the Internet, and can also offer self-service network access [3][4]. Services provided by Cloud Computing to users based on the resource through the virtual servers where users can access wherever they are and any detail that they need [5]

\section{1) Cloud Computing Services}

Cloud Computing services support a variety of technical functions. The services provided by Cloud are divided into three service models [3], namely Software as a Service (SaaS), Infrastructure as a Service (IaaS) and Platform as a Service (PaaS).

The architecture and services of cloud computing is shown in Fig. 1.

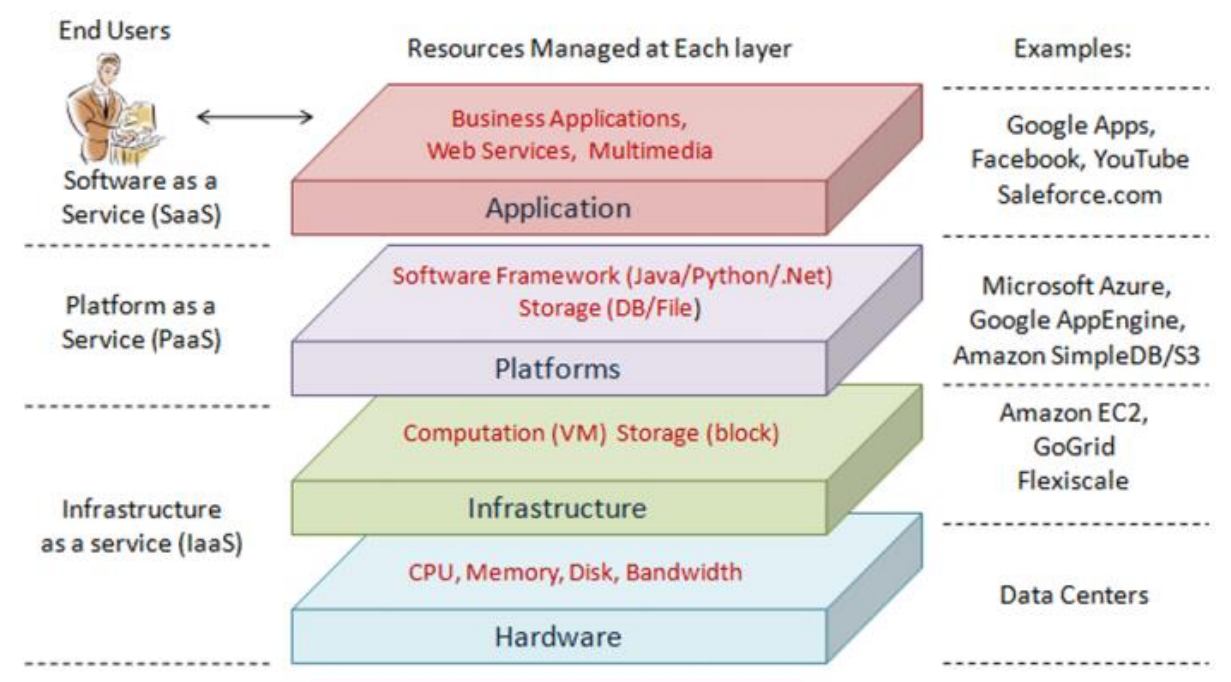

Fig.1. The Architecture of Cloud Computing [4]

\section{2) The Advantages of Cloud Computing for Healthcare Applications}

Cloud Computing offers a variety of advantages for the users such as less required power for the information systems, an increase in efficiency of data center operations, and lower operating costs [7]. Cloud Computing-based healthcare applications are benefited from Cloud Computing in the following ways [8][9]:

- Patient Security \& Privacy: Cloud offering enhanced security by utilizing Private Cloud to prevent leakage of patient's data and other sensitive health-related procedure.

- Scalability

- Dynamic Sensor Data Rates: The scalability of cloud architecture allows it to capture a variety of non-deterministic data flow.

- Global Access and availability

- Disaster Recovery, Reliability, and Redundancy

- Economical System Usage

- Outsourcing Expertise

\section{Internet of Things (IoT)}

IoT is a fairly new concept in terms of connecting several objects in a smart way. IoT is an intelligent technology that opens new development opportunities for service providers. IoT was first introduced by Kevin Ashton in 1999 and can be used to identify, locate, track and monitor objects automatically and in real-time. IoT is increasingly becoming an ecosystem that connects billions of intelligent objects [10], offers intelligent electrical measuring instrument readings, monitoring telemedisin, green house monitoring, and intelligent transportation using computer technology [11].

\section{Bluetooth}

Bluetooth is designed for close-range transmission and it is implemented on cheap devices to replace mouse, keyboard, joystick, and printers. Bluetooth has two types of topology, which are piconet and scatternet. A Bluetooth device can participate in multiple piconets at the same time, while allowing information to flow to an area outside of the piconet. A device in a scatternet can be a slave in several piconets, but there is only one master/main device among them [12].

\section{E. Wirelesss Fidelity}

Wireless fidelity (Wi-Fi) standard includes IEEE $802.11 \mathrm{a} / \mathrm{b} / \mathrm{g} / \mathrm{n}$ for wireless local area networks (WLAN), and it allows users to browse the Internet at broadband speeds while connected to an access point (AP) or in ad hoc mode [12]. 


\section{F. GPRS/UMTS}

\section{1) $G P R S$}

GPRS is an important technology of always-on data connection for applications such as web browsing and Push-to-Talk via mobile cell [13].

\section{2) UMTS}

The third generation of cellular technology that is developed by 3GPP, also known as Universal Mobile Telecommunications System (UMTS), Freedom of Mobile Multimedia Access (FOMA), 3GSM, etc., is a technology based on radio technology Wideband-Code Division Multiple Access (W-CDMA), which offers higher spectrum efficiency and bandwidth than GSM [14].

\section{WiRELESS Health MONITORING Systems (WHMS)}

In the past decade, WHMS has attracted the attention of the researcher community as well as the industrial world. Various numbers of research and development have been published in scientific papers. In this paper, six WHMS with different communication media such as Bluetooth, Wi-Fi, or GPRS/UMTS are analyzed.

\section{A. Ubiquitous Mobile Health Monitoring System for} Elderly (UMHMSE)

UMHMSE is designed to monitor the movement, location, and health status of the patient. UMHMSE monitor elderly patients from both the outside and inside the patient's environment. UMHMSE uses a bio sensor, which is worn by the patient, and a Smartphone as a center node. The data is collected and sent to the server via GPRS/UMTS to be analyzed further. The system monitors the patient's vital signs such as heart rate from a far distance. UMHMSE is also able to provide real-time alerts to family members or their doctors when the health statuses of the patients worsen [15]. UMHMSE has the following three components:

\section{- Wireless Wearable Body Area Network (WWBAN)}

WWBAN includes several sensors that are tailored to the body of the patient and these sensors obtain the relevant data and transfer the data to the Intelligent Central Node (ICN) by using Bluetooth. From Fig. 2 it can be seen that WWBAN forms a star topology that is centralized on the core sensor [15].

\section{- Intelligent Central Node (ICN)}

The duty of ICN is to gather and process the data that is sent by WWBAN and ICN is operated by communicating with Intelligent Central Server (ICS) using GPRS/UMTS on a smartphone. The smartphone is usually already embedded with Global Positioning System (GPS) feature to provide a mobile monitoring system. The flow diagram of how WWBAN, ICN, and ICS works is shown in Fig. 3 [15].

From Fig. 3 It can be seen that the sensors collect data from the WWBAN, accelerometer (the position of the patient), and Location Area Identifier namely Mobile Country Code (MCC), Mobile Network Code (MNC), Location Area Code (LAC) and Cell Identification (CI). The data will be sent to ICS if there is no change in the collected data.

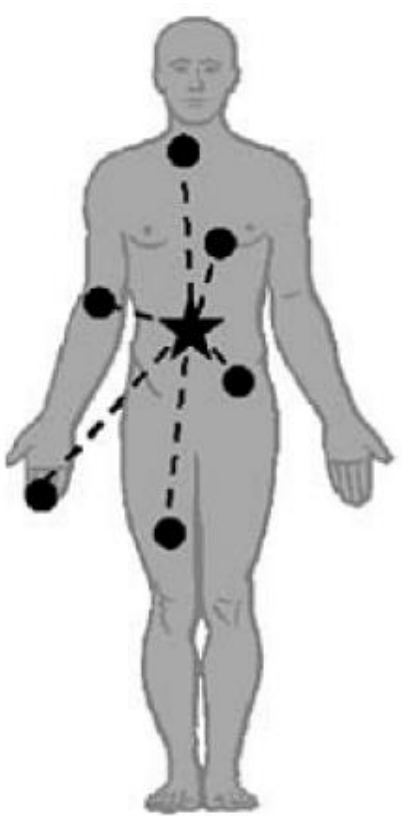

Fig.2. Topology Star-based WWBAN [15]

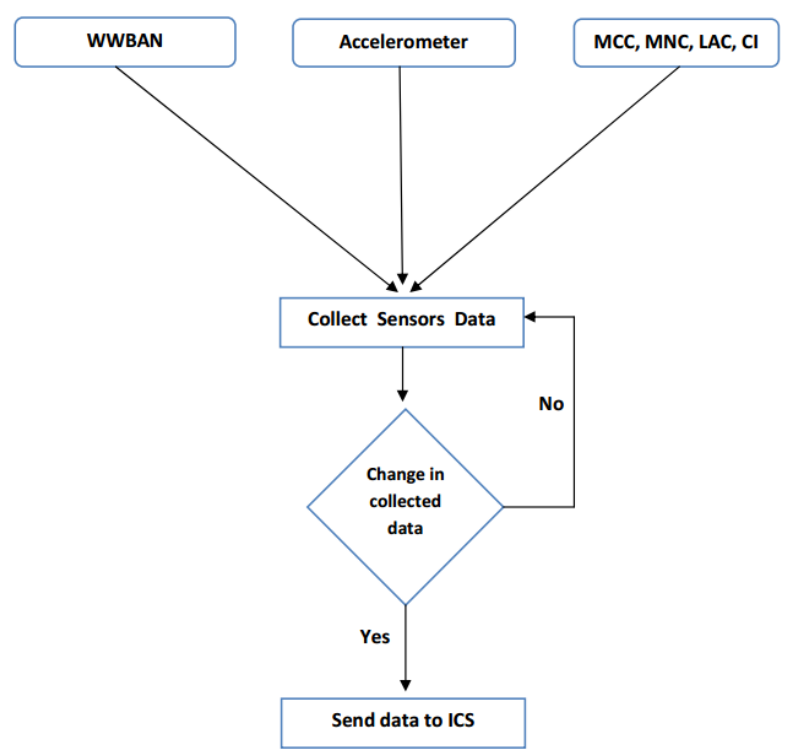

Fig.3. Flow Diagram of INC [15].

\section{- Intelligent Central Server (ICS)}

As mentioned earlier, ICN sends all the data to ICS to be analyzed separately without intervention from medics or doctors. Logistic regression technique is used in ICS to gather data and foresee the patient's health risk from the patient's mobility history. The health professionals as well as the patient's family will be informed about the health status of the patient once the data has been processed, if there is an unusual situation, the health 
status and the location of the patient will be informed to both medics and patient's family [15].

A prototype for UMHMSE has been implemented and Nonin 4100 Bluetooth Pulse Oximeter is used in the WWBAN as its sensor. The smartphone that is used for ICN is Nokia N95 with Symbian OS v. 9.2, while for the ICS, MySQL database and Apache web server are used.

Though having various advantages, UMHMSE also has a disadvantage, which is the phone battery capacity cannot handle UMHMSE in a long time due to the large number of the data, therefore, new technique to reserve the power consumption is needed.

\section{B. SHIMMER-based Windows mobile system}

This system consists of a SHIMMER-based network sensor body that is used to measure and collect the parameters of the patients. Communication between the sensor and mobile devices is done via Bluetooth connection. The system consists of the following three modules [16]:

- Sensors body that includes the body temperature sensor, sensor electrocardiography (ECG), sensor electroencephalography (EEG), electromyography sensors (EMG), a blood pressure sensor (NIBP), and a motion sensor.

- Processor unit that is responsible for obtaining and collecting the parameters that are measured in the long term.

- Windows Mobile devices, used for collection, display and control of the values measured.

Fig. 4 illustrates the architecture of the SHIMMERbased WHMS.

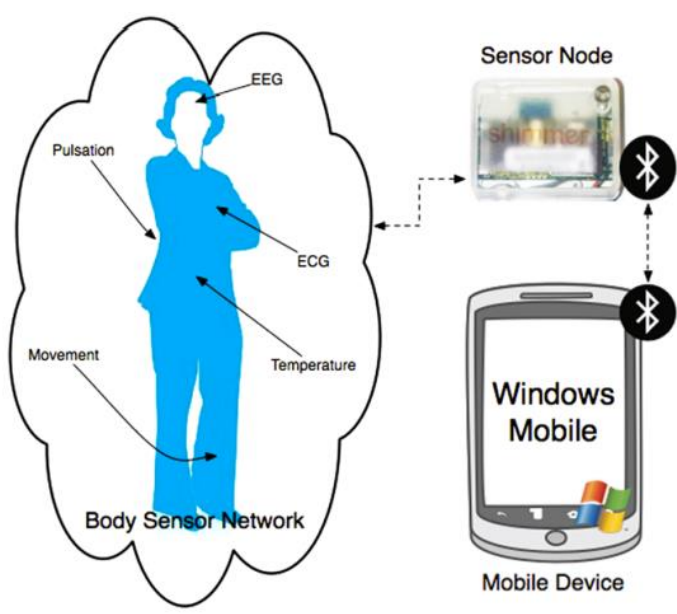

Fig.4. SHIMMER-based WHMS Architecture [16].

Almost all smartphone consumes the same level of power when a Bluetooth is used as its medium. From several tests, it can be concluded that Bluetooth drained the battery for $4 \%$ to $6 \%$, and it is considered efficient [16].

Three smartphone have been tested and compared, Table 1 presents the results in five different parameters.
- Initialization time. This parameter shows the time that is needed for the smartphone to start the system.

- Connection time. This is the time needed to connect to the Body Sensor Network (BSN).

- Graphical presentation. The time that is needed to produce the graphical data presentation.

- Smoothness. This parameter shows smoothness of the application with $1 \%$ being the worst and $100 \%$ being the best.

- Percentage of corrupted data

Table 1. SHIMMER-based WHMS Performance Results

\begin{tabular}{|c|c|c|c|}
\hline & HP iPAQ & HTC HD & $\begin{array}{c}\text { Asus } \\
\text { M39W }\end{array}$ \\
\hline $\begin{array}{c}\text { Initialization time (in } \\
\text { seconds) }\end{array}$ & $6 \mathrm{~s}$ & $3 \mathrm{~s}$ & $5 \mathrm{~s}$ \\
\hline Connection (in seconds) & $2.2 \mathrm{~s}$ & $2 \mathrm{~s}$ & $2.1 \mathrm{~s}$ \\
\hline $\begin{array}{c}\text { Graphical presentation } \\
\text { (in second) }\end{array}$ & $1.5 \mathrm{~s}$ & $1 \mathrm{~s}$ & $1.5 \mathrm{~s}$ \\
\hline $\begin{array}{c}\text { Smoothness (percent) } \\
\text { Percentage of computed } \\
\text { data (300 s) }\end{array}$ & $75.00 \%$ & $90.00 \%$ & $80.00 \%$ \\
\hline
\end{tabular}

Hard-coded and unique passwords as well as the use of specific connection port are used to protect the BSN and all of its nodes from the outside interference. This password is required to pair the device and give access to the data. Fig. 5 shows the hardware of the SHIMMERbased WHMS.

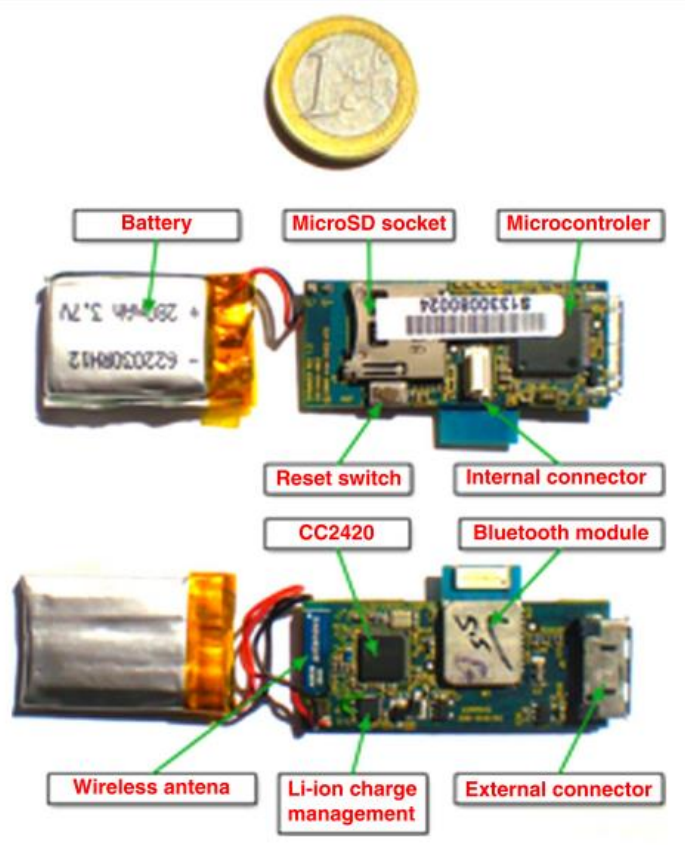

Fig.5. The Hardware of SHIMMER-based WHMS [16].

\section{Multi-Hopping Wireless Body Area Network system (WBAN)-based WHMS}

This system uses a medical bracelet to get the parameters of the patients from the sensor nodes. Medical bracelet is selected to reduce the interference that might 
occur between the sensor devices and network devices. This system uses medical wireless gateway to improve the operating area of multi-hopping, moreover, this gateway is used to collect sensor nodes to a LAN or the Internet. By using the Internet, the patient's healthcare professionals can access the patient physiological data from anywhere and at any time [17]. The system architecture is illustrated in Fig. 6.

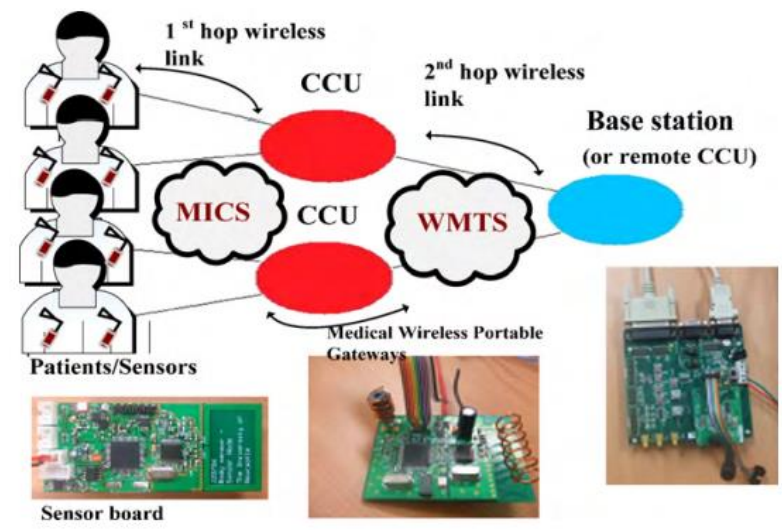

Fig.6. The System Architecture for Multi-Hopping WBAN-based WHMS [17].

This system uses polling mechanism-based sensor nodes that consume less power than Carrier Sense Multiple Access with Collision Avoidance (CSMA/CA)based nodes. With polling mechanism-based sensor, the application can manage the sleep and wake up mode, thus, increasing the battery life (up to several years), whereas, with CSMA/CA-based nodes are always active, even when it waits the Acknowledgement (ACK) packets. Battery life for CSMA/CA-based sensor only lasts up to three days.

Different range and frequency to detect and transmit the physiological signals are used in this system as shown in Table 2.

Table 2. Range and Frequency for Physiological Signals [17]

\begin{tabular}{|c|c|c|}
\hline Parameter & $\begin{array}{c}\text { Range of } \\
\text { parameter }\end{array}$ & $\begin{array}{c}\text { Signal frequency } \\
(\mathrm{Hz})\end{array}$ \\
\hline ECG Signal & $0.5-4 \mathrm{mV}$ & $0.01-250$ \\
\hline Respiratory rate & $2-50$ breaths/min & $0.1-10$ \\
\hline Blood pressure (BP) & $10-400 \mathrm{mmHg}$ & $0-50$ \\
\hline EEG & $3-300 \mu \mathrm{V}$ & $0.5-60$ \\
\hline Body temperature & $32-40^{\circ} \mathrm{C}$ & $0-0.1$ \\
\hline $\begin{array}{c}\text { EMG } \\
\text { (electromyogram) }\end{array}$ & $10 \mu \mathrm{V}$ to $15 \mathrm{mV}$ & $10-500$ \\
\hline $\begin{array}{c}\text { GSR (galvanic skin } \\
\text { reflex) }\end{array}$ & $30 \mu \mathrm{V}$ to $3 \mathrm{mV}$ & $0.03-20$ \\
\hline
\end{tabular}

Signals from the patient, which usually mixed with noise, are collected by the sensor node. These signals are converted to digital by using the Analog to Digital Converter (ADC) to be processed digitally and stored in a microcontroller. The processed signals are transmitted via a wireless transmitter. The sensor nodes are draining less battery life because it small and power efficient.

\section{Hybrid Mobile-Cloud Approach for ECG} Telemonitoring

This WHMS is a solution to deal with restrictions on the monitoring and managing the health status of patients [18]. This system's test results have shown that the system can significantly improve the conventional health monitoring system in diagnosing and providing medical action. Fig. 7 illustrates the ECG Telemonitoring using Hybrid Mobile Cloud.

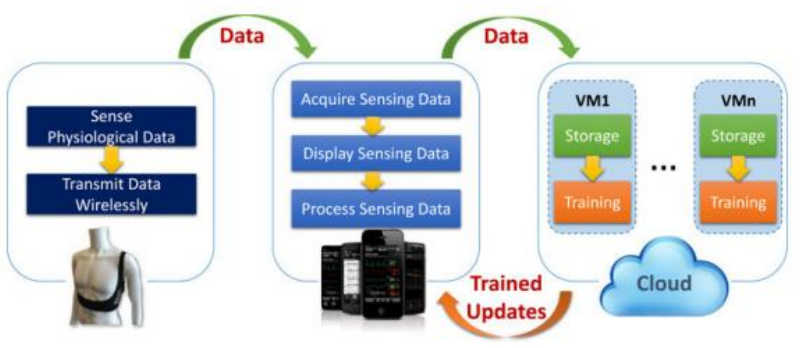

Fig.7. Architecture of ECG Telemonitoring using Hybrid Mobile Cloud [18]

Prototype for this system has been implemented on a Google Galaxy Nexus with Android OS v. 4.2. The heartbeats signal are detected and extracted by processing the incoming ECG data, furthermore, this signal is forwarded to the processing engine that is based on Artificial Neural Network (ANN). When the user launches the application, the ECG data of the patients as well as the diagnostic from his medics are sent to the server in cloud network. Two individual execution threads were implemented to ensure a continuous monitoring, these threads are as follows: Thread 1 handles the data that are transmitted to the cloud, while Thread 2 process the ECG signals with the ANN-based processing engine [18].

Although this system has been able to perform well and proven to be able to reduce the power consumption, there are some issues that might occur such as security, the decreasing of the accuracy of diagnosis and calculating performance and it has limited computational power. Intensive data communications between the mobile devices and the cloud can lead to high level security threats, which must be addressed as soon as possible.

\section{E. BLE-based Wireless Electrocardiogram Monitoring System}

BLE-based Wireless Electrocardiogram Monitoring System is proposed by $\mathrm{Yu}$ et al. [19]. This system consists of three following components:

- Single-chip ECG signal acquisition module

- Bluetooth module

- Smartphone

This system is able to acquire signals through sensors ECG 3-lead or 12-lead electrocardiography (ECG), moreover, the system can also transmit data via Bluetooth wireless ECG link to a smartphone for further processing 
and displaying the ECG signals. The system's tests results show that the system can run in a long period of time in accordance with the BLE technology. Fig. 8 depicts the architecture of this system.

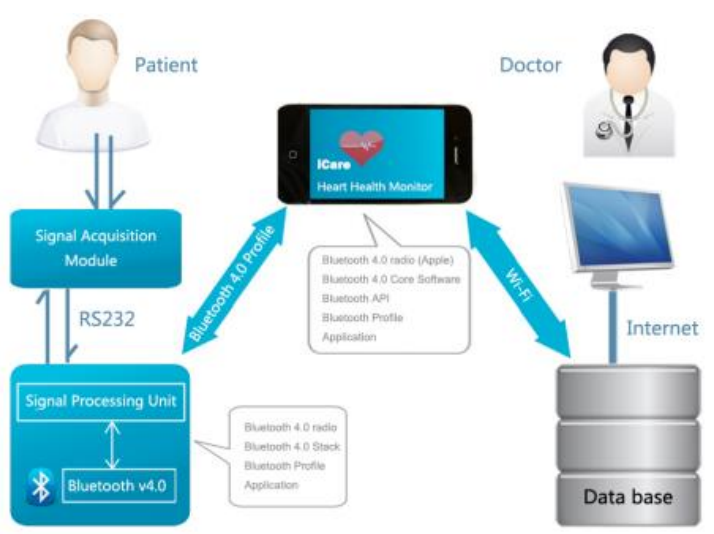

Fig.8. System Architecture for BLE-based WHMS [19]

Important features of this system are the following; the system uses less power than the other wireless medical devices. In addition, the system is able to detect and process the ECG data and QRS information on smartphone to help patients and medical personals. This system greatly improved mobility, flexibility, and the use of ECG monitoring systems for health care and the BLE as its medium communication reduces its power usage.

A single-chip acquisition system is used to measure the patient's ECG, it filters the high frequency interference that is included in the signal. The ECG signals are transmitted to the RS232 at 250 samples per second. A power management logic controls is included in the ECG signal acquisition module, and it only obtain and transfer the patient's data when he contacts all three electrodes. The circuit to detect the contact will wake up the smartphone when the impedance is smaller than $5 \mathrm{M} \Omega$, the delay for wake up process is below $5 \mathrm{~ms}$. The sleep mode is entered when the input impedance rises above 9 $\mathrm{M} \Omega$ for more than $300 \mathrm{~ms}$ [19].

The main advantage of this system is that it uses less power than the other WHMS. Moreover, this system is able to process the ECG data and detect the QRS information on the patient's smartphone. BLE technology that is used in this device improves the system's mobility and flexibility, and it can be used not only for monitoring the patient's condition, but also ambulatory and rehabilitation monitoring.

Fig. 9 illustrates the graphical view of the ECG signal on the patient's smartphone. User can press the "record" button to save the data in a text file or user can press the "mailing" button to transfer the ECG text file via e-mails. The data transmission is done by using the Wi-Fi.

\section{F. Phone-based e-Health System for Obstructive Sleep Apnea Syndrome (OSAS)}

A system to monitor respiratory disease called OSAS is presented by Gao et al. [20]. The disease does not only interrupt normal sleep but also leads to hypoxemia and hypercapnia. The system is very efficient in terms of power consumption due to the use of Bluetooth technology. Fig. 10 illustrates the architecture of the system.

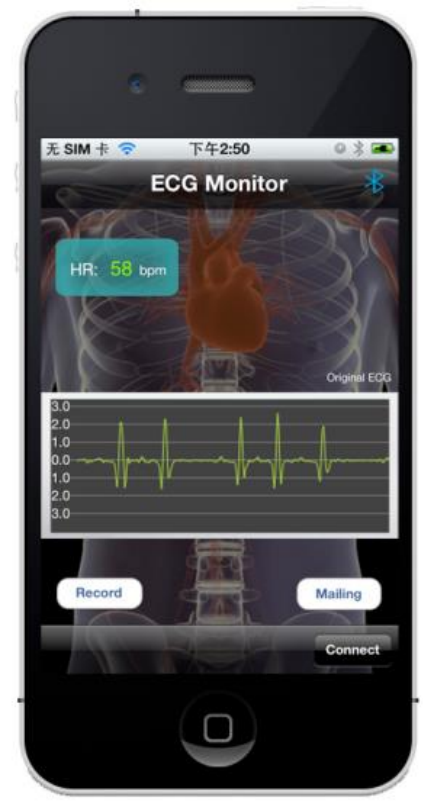

Fig.9. ECG Signal Displayed on Smartphone [19]

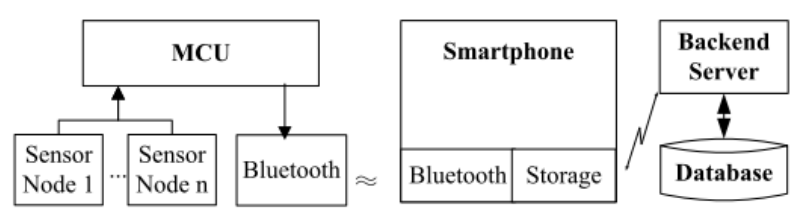

Fig.10. System Architecture for OSAS Monitoring [20]

It can be seen in Fig. 11 that this system consists of several components as follows:

- Two sensor nodes to transmit vital signs ( $\mathrm{SpO} 2$ (oxygen saturation in the blood) index and ECG)

- A micro-control unit (MCU) to perform data processing

- A smartphone to diagnose the data

- A backend server to provide health services more sophisticated

The MCU is crucial in this system as it uses the power more efficiently. The signal noise reduction process and the signal encoding process that come from the raw data must be dealt carefully. During the experiment, this system proved that the MCU duration was more than 20 hours, while the smartphone was only for 6 hours. The MCU is chosen because it is light-weight, consumes less power, and easy to learn, moreover, the requirements of OSAS does not require a more capable equipment than MCU.

The prototype was design as small and as portable as possible by choosing Arduino Pro Mini MCU, Samsung I9100 with Android OS was used and the Bluetooth version was GC-02. The battery that was used is a $3.3 \mathrm{~V}$ battery. Table 3 presents the hardware that is used in OSAS. 
Table 3. Hardware used in OSAS [20]

\begin{tabular}{|c|c|c|}
\hline \multicolumn{2}{|c|}{ Module } & Type \\
\hline \multirow{4}{*}{ Frontend } & MCU & Arduino Pro Mini \\
\cline { 2 - 3 } & Bluetooth & $\begin{array}{c}\text { GC-02From Nanjing Guochun } \\
\text { Tech Inc. }\end{array}$ \\
\cline { 2 - 3 } & $\mathrm{SpO}_{2}$ Sensors & $60 \mathrm{~Hz}$ sampling rate \\
\cline { 2 - 3 } & ECG Sensor & One-Lead, 250Hz sampling rate \\
\cline { 2 - 3 } & Battery & $1200 \mathrm{mAh}$ Li Battery \\
\hline \multicolumn{2}{|c|}{ Smart Phone } & I9100 with Android OS 2.3.4 \\
\hline \multicolumn{2}{|c|}{ Backend Server } & PC \\
\hline
\end{tabular}

It can be seen in Table 4 that the average power consumption is $187.8 \mathrm{~mW}$, this means that the power consumption is about $676 \mathrm{~J} /$ hour, and moreover, for 1200 mAh battery, the system can last up to 21 hours.

Table 4. Comparison of Each Module in term of Power Consumption

\begin{tabular}{|c|c|c|}
\hline Module & State & Power Consumption $(\boldsymbol{m} \boldsymbol{W})$ \\
\hline MCU & Active & 29.16 \\
\hline \multirow{2}{*}{$\begin{array}{c}\text { Bluetooth } \\
\text { module }\end{array}$} & Idle & 19.80 \\
\cline { 2 - 3 } & Active & 105.4 \\
\hline Sensor & Active & 53.16 \\
\hline Frontend Total & 5m Distance & 187.7 \\
\hline
\end{tabular}

There are active and idle mode for the Bluetooth module and the Bluetooth module consumes $105.4 \mathrm{~mW}$ in active mode and $19.8 \mathrm{~mW}$ in idle mode. Idle mode consumes less power and it can be used to reduce the power consumption of the whole system, thus, the system is programmed to enter the idle mode regularly. Implementation of this system is illustrated in Fig. 11 [20].

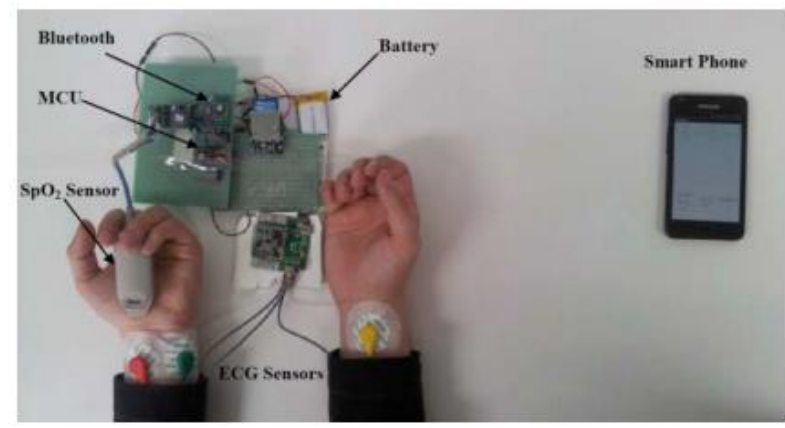

Fig.11. The Implementation of OSAS [20].

In order to improve the power consumption issue, the following suggestions can be made:

1. The Bluetooth module can be programmed to enter the idle mode periodically so that the power can be saved. Moreover, to prevent the data loss in the idle mode, an extra memory to store the incoming data should be made.

2. Longer idle time can be used for more power saving, however, the power saving cannot repay the cost for having an extra memory, therefore, the usage between idle time an extra memory should be balanced.

\section{ANALYSIS ON POWER EFFICIENCY}

From the description of six WHMS in Section 3, the table to compare each system can be made as follows:

Table 5. WHMS Comparison

\begin{tabular}{|c|c|c|c|}
\hline System & $\begin{array}{c}\text { Medium } \\
\text { Communication }\end{array}$ & Features & $\begin{array}{c}\text { Efficient } \\
\text { use of } \\
\text { power }\end{array}$ \\
\hline $\begin{array}{l}\text { UMHMSE } \\
\text { [15] }\end{array}$ & GPRS/UMTS & Telemedicine & No \\
\hline $\begin{array}{l}\text { SHIMMER- } \\
\text { based } \\
\text { Windows } \\
\text { mobile } \\
\text { system [16] }\end{array}$ & Bluetooth & & Yes \\
\hline $\begin{array}{c}\text { Multi- } \\
\text { Hopping } \\
\text { WBAN- } \\
\text { based } \\
\text { WHMS [17] }\end{array}$ & Bluetooth, Wi-Fi & $\begin{array}{l}\text { ECG, NiBP, } \\
\text { Pulse, EEG }\end{array}$ & Yes \\
\hline $\begin{array}{c}\text { Hybrid } \\
\text { Mobile- } \\
\text { Cloud } \\
\text { Approach for } \\
\text { ECG } \\
\text { Telemonitori } \\
\text { ng [18] }\end{array}$ & Wi-Fi & ECG & No \\
\hline $\begin{array}{l}\text { BLE-based } \\
\text { Wireless } \\
\text { ECG } \\
\text { Monitoring } \\
\text { System [19] }\end{array}$ & Bluetooth & ECG & Yes \\
\hline $\begin{array}{l}\text { Phone-based } \\
\text { e-Health } \\
\text { System for } \\
\text { OSAS [20] }\end{array}$ & Bluetooth & $\begin{array}{l}\text { ECG, NiBP, } \\
\text { Pulse }\end{array}$ & Yes \\
\hline
\end{tabular}

From the comparison of six WHMS as shown in Table 5 , the systems that run remotely, such as the UMHMSE, do not use power efficiently, this is due to the use of GPRS/UMTS as its medium communication. GPRS/UMTS requires large power to be connected to the wireless network continuously. This is in contrast with the Multi-Hopping WBAN-based WHMS and BLE-based Wireless ECG monitoring system that uses Bluetooth connections which proved very efficient in power use. However, the Multi-Hopping WBAN-based WHMS use power more efficiently in terms of capacity, features and coverage of services as it can detect the ECG, NIBP, Pulse, and EEG data, compared with BLE-based Wireless ECG monitoring system that only has the features of monitoring the ECG data.

It can be seen in Table 1 that Bluetooth as a medium communication is a low cost technology and consumes less power than other technologies. All six WHMS that are analyzed in this paper provide enough security as it authenticates all devices that can receive, process, and display the data. 


\section{CONCLUSIONS}

The integration of Cloud Computing and IoT has been an attention of researchers all over the world in recent years. Cloud Computing is used to provide Software as a Service (SaaS) by connecting smart objects via the Internet. With Cloud Computing stores different data, the IoT provides systematic commands that can be run on any devices that are connected to the internet. Therefore, it can be concluded that Cloud Computing that is integrated with the IoT is very useful, and the resulting performance greatly assist patients and health professionals to monitor the condition of the patient's body and disease.

Further conclusion is, the communication between the sensor nodes of WHMS and mobile devices that is done by using Bluetooth (BLE) has proven effective in reducing power consumption compared with other technologies (GPRS/UMTS or Wi-Fi). Moreover, the latest version of Bluetooth technology will be able to monitor medical condition at even lower power consumption.

\section{FUTURE WORK}

This paper is an initial part of our research, which is to propose a new WHMS that is more efficient in power use and able to detect monitor more health parameters, moreover, the new WHMS should be able to provide more security to prevent any threats. As a medium communication, BLE has been analyzed to be able to reduce power consumption, however, different medium communication can also be used, such as the newly introduced Li-Fi (Light-Fidelity) that uses Light Emitting Diode (LED) to transmit and receive data. $\mathrm{Li}-\mathrm{Fi}$ is promising because LED is practically used in almost everywhere, and it is proven to be a lot faster than Bluetooth and Wi-Fi.

\section{ACKNOWLEDGMENT}

The authors wish to thank all students and colleagues of the Security ICT groups of Mercu Buana University that encouraged and helped us to do our research.

\section{REFERENCES}

[1] K. Ahmed and M. Gregory, "Integrating wireless sensor networks with cloud computing," in 2011 Seventh International Conference on Mobile Ad-hoc and Sensor Networks, 2011, pp. 364-366.

[2] E. Troubleyn, I. Moerman, and P. Demeester, "QoS Challenges in Wireless Sensor Networked Robotics," Wirel. Pers. Commun., vol. 70, no. 3, March. 2013,pp. 1059-1075,.

[3] P. Mell and T. Grance, "The NIST Definition of Cloud Computing-Recommendations of the National Institute of Standards and Technology NIST," NIST Spec. Publ., 2011.

[4] Qi Zhang, Lu Cheng, and Raouf Boutaba, "Cloud computing: state-of-the-art and research challenges," Springer J Internet Serv Appl (2010) 1: 7-18.
[5] S. Rajan and A. Jairath, "Cloud Computing: The Fifth Generation of Computing," in 2011 International Conference on Communication Systems and Network Technologies, 2011, pp. 665-667.

[6] M. Yuriyama and T. Kushida, "Sensor-cloud infrastructure-physical sensor management with virtualized sensors on cloud computing," in 13th International Conference on Network-Based Information Systems, 2010, pp. 1-8.

[7] ENISA, "Security and Resilience in Governmental Clouds," 2011.2 [Online]. https://www.enisa.europa.eu/publications/security-andresilience-in-governmental-clouds. [Accessed: 03-Jul2016].

[8] R. Aiswarya, R. Divya, D. Sangeetha, and V. Vaidehi, "Harnessing healthcare data security in cloud," in 2013 International Conference on Recent Trends in Information Technology (ICRTIT), 2013, pp. 482-488.

[9] Rao, B.B.P.; Saluia, P.; Sharma, N.; Mittal, A.; Sharma, S.V., "Cloud computing for Internet of Things \& sensing based applications," in 2012 Sixth International Conference on Sensing Technology (ICST), 2012, pp. 374-380.

[10] S. Ziegler, C. Crettaz, L. Ladid, and S. Krco, "Iot6moving to an ipv6-based future iot," vol. 7858, pp. 161172, 2013.

[11] H. Zhang and L. Zhu, "Internet of Things: Key technology, architecture and challenging problems," 2011 IEEE Int. Conf. Comput. Sci. Autom. Eng., pp. 507-512, Jun. 2011.

[12] Jin-Shyan Lee, Yu-Wei Su, and Chung-Chou Shen (2007), "A Comparative Study of Wireless Protocols: Bluetooth, UWB, ZigBee, and Wi-Fi", The 33rd Annual Conference of the IEEE Industrial Electronics Society (IECON), Nov. 5-8, 2007.

[13] ETSI, "General Packet Radio Service (GPRS)," 2016. [Online]. Retrieved from: http://www.etsi.org/technologiesclusters/technologies/mobile/gprs. [Accessed: 03-Jul2016].

[14] ETSI, "Universal Mobile Telecommunications System (UMTS)," $2016 . \quad$ [Online]. Retrieved from: http://www.etsi.org/technologies-

clusters/technologies/mobile/umts. [Accessed: 03-Jul2016].

[15] Bourouis, A., Feham, M., and Bouchachia, A.(2011), " Ubiquitous Mobile Health Monitoring System for Elderly (UMHMSE)", International Journal of Computer Science and Information Technology, Vol.2, No. 3, June, pp. 74-82

[16] Orlando R. E. P., Caldeira, M. L. P. Lei S., and Rodrigues, J.P.C (2014), "An Efficient and Low Cost Windows Mobile BSN Monitoring SystemBased on TinyOS", Journal of Telecommunication Systems, Vol. 54, No. 1, pp. 1-9

[17] Yuce, M. R.(2010)" Implementation of wireless body area networks for healthcare systems", Sensor and Actuators A:Physical, Vol. 162, No. 1, July, pp. 116-129

[18] Xiaoliang Wang; Qiong Gui; Bingwei Liu; Zhanpeng Jin et al (2014), "Enabling Smart Personalized Healthcare: A Hybrid Mobile-Cloud Approach for ECG Telemonitoring", IEEE Journal of Biomedical and Health Informatics, Vol. 18, No. 3, May, pp. $739-745$

[19] Bin Yu; Lisheng Xu; Yongxu Li (2012) "Bluetooth Low Energy (BLE) based mobile electrocardiogram monitoring system", In the proceedings of International Conference on Information and Automation, June 6-8, 
2012, Shenyang, pp. 763-767 International Journal of Computer Networks \& Communications (IJCNC), Vol.7, No.3, May 201529

[20] Ruipeng Gao; Liqiong Yang; Xinyu Wu; and Tao Wang, (2012) "A phone-based e-health system for OSAS and its energy issue", In the proceedings of the International Symposium on Information Technology in Medicine and Education, August 3-5, 2012, Hokodate, Hokkaido, pp. 682-696

[21] G. Suciu, A. Vulpe, S. Halunga, O. Fratu, G. Todoran, and V. Suciu, "Smart Cities Built on Resilient Cloud Computing and Secure Internet of Things," in 201319 th International Conference on Control Systems and Computer Science, 2013, pp. 513-518.

\section{Authors' Profiles}

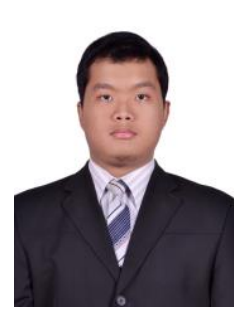

Beny Nugraha was born in Jakarta in 1989. $\mathrm{He}$ received his B.Sc degree in Telecommunication Engineering from Bandung Institute of Technology, Indonesia, and Double Degree-M.Sc in Telecommuniation Engineering from Bandung Institute of Technology, Indonesia, and Hochschule Darmstadt, Germany. Currently, he is a lecturer in Electrical Engineering Department at Mercu Buana University in Jakarta, Indonesia. His research focus on network security, and currently he is focusing on the security of Future Internet Architectures and mobile application.

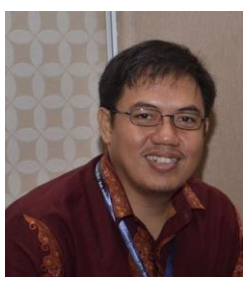

Irawan Ekasurya was born in 1974 in Indonesia. He received Bachleor degree in information technology from STMIK Jayakarta, Indonesia and a Master student at electrical engineering, Mercu Buana University, Jakarta, Indonesia. His research interests are Wireless Sensor Network, Internet of Things, Data Mining,

Big Data, eHealthcare.

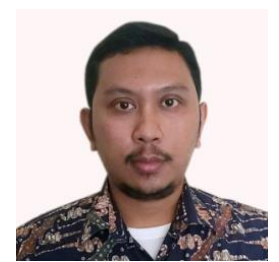

Gunawan Osman was born in 1982 in Indonesia. He received Amte degree in Biomedical Academi from Biomedical Academi Andakara, Indonesia and ST degree in Electronica Engineering from Institute Sains and Technology, Indonesia. Since 2003, he is Engineer in Radjak Group Corporate. His research interest are Network Security, Biomedical technology, Digital Forensic.

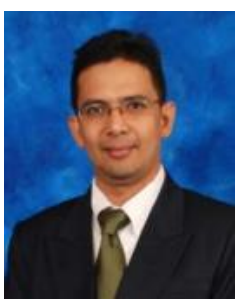

Mudrik Alaydrus was born in Jakarta in 1971. He received his Dr.-Ing degree in Electrical Engineering (Computational Electromagnetics) from Wuppertal University, Germany. Currently, he is a Professor of Telecommunication and Electromagnetics in Department of Electrical Engineering, Universitas Mercu Buana UMB, Jakarta. His research focus Computational Electromagnetics, Wireless Systems, Signal
Processing, and Mathematical Modeling.

How to cite this paper: Beny Nugraha, Irawan Ekasurya, Gunawan Osman, Mudrik Alaydrus,"Analysis of Power Consumption Efficiency on Various IoT and Cloud-Based Wireless Health Monitoring Systems: A Survey", International Journal of Information Technology and Computer Science(IJITCS), Vol.9, No.5, pp.31-39, 2017. DOI: 10.5815/ijitcs.2017.05.05 